\title{
Efficacy and safety of oral solution dosed misoprostol versus misoprostol vaginally in labour induction*
}

\author{
Longinos Aceituno Velasco, María Teresa Sánchez Barroso, María Huertas Segura García, \\ Valois González Acosta, Ramón de la Fuente Pedrosa, Ana Barqueros Ramírez, \\ Luis Delgado Martínez, Encarna Ruiz Martínez
}

Gynaecologic Clinic Management Unit, Hospital “La Inmaculada” (Huercal-Overa), Healthcare Management Area North of Almeria, Andalusian Health Service, Andalusian, Spain

Email: laceitunov@sego.es, longinos.aceituno.sspa @ juntadeandalucia.es

Received 3 May 2013; revised 2 June 2013; accepted 10 June 2013

Copyright (c) 2013 Longinos Aceituno Velasco et al. This is an open access article distributed under the Creative Commons Attribution License, which permits unrestricted use, distribution, and reproduction in any medium, provided the original work is properly cited.

\begin{abstract}
Background: Labour induction is one of the most common medical procedures in obstetrics. The aim is to end the pregnancy when continuity is a risk to mother or fetus. Its main side effect is the increase in the cesarean rate, compared to spontaneous onset deliveries. On the other hand, mortality and morbidity in cesareans are higher. The most common pharmacological drugs used for induction are prostaglandins: dinoprostone and misoprostol. The "gold standard" for labour induction is vaginal misoprostol. The oral route is also effective and also has several benefits like faster onset and easear administration. In recent years several publications state that the administration of misoprostol oral solution, given in doses gradually, is associated with a lower cesarean and hyperstimulation rate than the cases where vaginal misoprostol has been used in pregnant women with unripe cervix. Furthermore, being its half life shorter, it may be very useful in case of uterine hyperstimulation and, probably, a high percentage of women prefer this oral administration to the vaginal one. The objective of this study is to compare the efficacy, safety and side effects on mother and fetus on use of oral versus vaginal administration for induction of labour for prolonged gestation (41 weeks) and premature rupture of membranes, both with live fetus. Methods/Design: Design: double blind controlled trial. Study population: Pregnant women whose labour will be induced due to premature rupture of membranes or prolonged gestation. Inclusion Criteria: 1) Bishop Test equal to or less than 7; 2) Single pregnancy; 3)
\end{abstract}

*Competing interests: The authors declare that they have no competing interests.
Pregnancy at term (37 - 42 weeks); 4) No history of uterine surgery; 5) Cephalic presentation; 6) Live fetus; 7) No prostaglandins contraindications. Discussion: Nowadays induction rates are very high, ranging from $25 \%$ to $30 \%$ approximately. In these cases caesarean rates are higher than when the delivery starts spontaneously. That is one of the main reasons why caesareans have increased, mainly in the cases of nuliparous women with immature cervix. If we can prove the hypothetical good results obtained through the use of dosed oral misoprotol, we will be able to reduce the number of induced deliveries by cesarean, and so improve the levels of security for the mother and the foetus, and, as a consequence, provide a higher quality of medical attention to the newborn and the mother.

Keywords: Cervical Ripening; Labour; Induction; Misoprostol; Administration; Oral; Administration; Intravaginal; Oxytocin; Dinoprostone; Pregnancy; Prolonged; Fetal Membranes; Premature Rupture

\section{BACKGROUND}

Induction of labour is one of the most common medical procedures in obstetrics. In the U.S. its frequency has increased steadily from $9.5 \%$ in 1990 , to $19.4 \%$ in 1998 [1], and $27 \%$ in 2003 [2].

Increase of the cesarean delivery rate is one of the main potential adverse effects of induction, compared with spontaneous onset of labour. That happens very often in the case of nulliparous women $[3,4]$ specially within those with unripe cervix [5-8].

The cost of surgery is $76 \%$ higher in cesarean delivery than that with vaginal birth, regardless of the readmission 
rate.

Ideally, the cesarean rate in the induced labours should be as similar as possible to the one of spontaneous deliveries. To obtain this outcome we should find the right drug and dosage to achieve these results.

The methods that have been used for labour induction have been numerous. If we just focus on drugs, we could mention, according to its importance, oxytocin and prostaglandins and within the latter dinoprostone and misoprostol. Oxytocin has the disadvantage of high failure rate when the cervix is immature.

\section{STATE OF THE ART}

Prostaglandins have been gaining ground to oxytocin in recent decades, mainly for preinduction/induction of labour, when the cervix is immature, but to choose between one or the other it is necessary to know which one works more efficiently and which one has got the safer maternal-fetal profile too.

Hofmeyr [9] in a review for the Cochrane that includes 121 clinical trials involving more than 10,000 women, finds out that vaginal misoprostol is more effective than dinoprostone and oxytocin for induction of labour (he gets higher rate of vaginal delivery within the first 24 hours, the differences being statistically significant), but rates of hyperstimulation and meconium are increased at doses higher than 25 mcg., but this does not result in higher rates of perinatal morbidity, although it is a motive of concern. The conclusion is that doses which exceed 25 mcg of misoprostol every 4 hours are more effective than conventional methods of cervical ripening, but they provoque greater rates of hyperstimulation. Doses below 25 mcg would have similar results and side effects.

Other data in favour of misoprostol are: it is cheap and it can be kept at room temperature, however dinoprostone is expensive and needs to be in a fridge.

Subsequent works such as Austin [10], Shakya [11] and Silfeler ones [12], published in 2010, also indicate that misoprostol is more effective (higher rate of vaginal delivery within the first 24 hours) than dinoprostone, and it also shows that fetal safety profile (no difference in the rate of hyperstimulation is established) is similar between the two of them.

Therefore, nowdays, the "gold standard" for cervical ripening and induction of labour is vaginal misoprostol at doses of 25 mcg every 4 - 6 hours [13-16].

Misoprostol may be administered by various routes: oral, vaginal, rectal and sublingual. In general, the vaginal route appears to be as effective as the other. However, the vaginal route seems to be associated with a higher incidence of uterine hyperstimulation than the rest of them, which can compromise fetal safety. This fact seems to be related to the dose provided.

The oral route is also effective and besides it has the benefit of the route of administration, which is better tolerated by women.

Alfirevic [17], in a meta-analysis for the Cochrane, having done 56 clinical trials with more than 11,000 women, finds out that, using oral misoprostol, the cesarean rate is lower than when administering dinoprostone; being the the rest of outcomes similar ones. When compared with vaginal misoprostol, no differences in the primary outcomes can be found, but there is a lower rate of infants with low Apgar. He concludes that oral misoprostol seems safer for the fetus than vaginal route and that doctors should use oral solution doses between 20 $25 \mathrm{mcg}$. To sum up, the oral route can improve clinical results of the vaginally one.

Obtaining a safe dose ranging below $50 \mathrm{mcg}$, just by dividing a misoprostol pill of $200 \mathrm{mcg}$, is inaccurate and therefore unreliable. It has been found out that only in $56 \%$ of the cases we would get the desired dose by dividing the pill into eight portions (if we want a $25 \mathrm{mcg}$ dose). This imprecision in the dosage would lead to insufficient or excessive dose that would cause an induction failure or a hyper-stimulation [18]. To improve the accuracy of dosage we can use an oral misoprostol solution. To get this solution we need to dissolve a misoprostol pill (200 mcg) in a beaker with 200 milliliters of water. In that way we would obtain a misoprostol solution concentration of $1 \mathrm{mcg} / \mathrm{ml}$. Furthermore, this solution would allow us to give exact misoprostol doses, as it happens with oxytocin, according to uterine response. This solution is stable for 24 hours and it dosage is more accurate than dividing up the misoprostol pill [19].

From a pharmacokinetic point of view, misoprostol in aqueous solution begins its action (4 minutes, range 2 - 5 min.) faster than if we use pills (6 min., Range 4 - $10 \mathrm{~min}$ ) or use the vaginal route (20 minutes), statistically significant and relevant differences $(p=0.01$ ) [20], although the serum level of misoprostol, and therefore its bioavailability, last longer with the vaginal administration [21]. The average uterine activity of the misoprostol oral solution within the first 15 minutes is bigger than if we administered it orally, rectally or vaginally and after these 15 minutes the activity declines gradually. Therefore, the effect of the aqueous solution will start earlier than with any of the other routes, but also the life span is shorter when compared with the oral or vaginal route, which can be an advantage, since the women's variable response to misoprostol and the possible risk of hyperstimulation [22].

Zieman [23] finds out that there is individual variability in the pharmacokinetic parameters of misoprostol absorption, but this variation is higher with vaginal misoprostol than with oral one, which may determine that if 
the reaction is different, the effectiveness may be affected too in the women induced vaginally. This may be another fact in favour of the oral route.

Based on these attractive pharmacokinetic characteristics for oral solution described above, several articles have come out in recent years analyzing this via. Moreover, some articles have used the attribute of this new track, which can be dosed according to uterine response.

Cheng [24] in 2008, performes a clinical trial with 207 pregnant women, comparing vaginal misoprostol 25 mcg every 4 hours until obtaining adequate dynamic with misoprostol solution dosed, beginning with $20 \mathrm{mcg} / \mathrm{hy}$ and progressively increasing, if no reply is obtained, to 40 and $60 \mathrm{mcg} / \mathrm{h}$. With the following results: $17 \%$ of caesarean sections in the vaginal misoprostol group and 4\% in the oral solution group (RR 0.20 95\% CI 0.07 to 0.62 ) and a rate of hyperstimulation of 12 and $0 \%$ respectively (RR0, 95\% CI 0.01 to 0.6108 ), although the study has been criticized afterwards due to the fact the lack of blindness in medication administration, the largest proportion of nulliparous in the vaginal misoprostol group (71.87\% vs $43.6 \%)$ and the exclusion of those pregnant women who requested epidural analgesia, a situation difficult to imagine nowadays.

Kundodyiwa [25] in 2009, performs a systematic review of the use of a dosed oral solution of misoprostol for labour induction compared with vaginal misoprostol, dinoprostone and oxytocin. He analyzes nine clinical trials involving more than 3,000 pregnant women. He finds a reduction in cesarean rate of $18 \%$ (RR 0.82, 95 confidence interval $0.71 \%$ to $0.96 \%$ ) and a lower incidence of uterine hyperstimulation with impact on the fetal monitor. He concludes his work by pointing out that oral misoprostol solution at low doses $(20 \mathrm{mcg})$ are equally effective, but it decrease the incidence of cesarean sections and uterine hieprestimulacion. He recommends a dose of $20 \mathrm{mcg}$ every 2 hours, with a maximum of 6 doses. He suggests a more continuous treatment scheme for the oral solution than for the vaginal route, because in the first case the effect remains less time. The results are similar comparing dinoprostone, vaginal misoprostol versus the dosed oral solution, but the doses used are lower (maximum $40 \mathrm{mcg} / \mathrm{h}$ ) than the ones that have been proposed later.

Souza [26] states, according to the results he obtained, that this new formulation is safe and effective and offers the following pattern: $20 \mathrm{mcg} / \mathrm{h}$ for 6 hours, increasing to 40 the following 6 hours, then to 60 for 6 hours more and finally administering 80, if no adequate dynamic is achieved; although $60 \%$ of pregnant women start delivery using doses of $20 \mathrm{mcg}$.

Shi-Yann Cheng [27] in 2010 performs a research to compare the results by parity. He finds out a cesarean rate of $8.2 \%$ in nulliparous women and $0 \%$ in multipa- rous ones, hyperstimulation rates are $0 \%$ for both groups. He suggests the following pattern: start administration with $20 \mathrm{mcg} /$ hour for the first four hours, if no response is obtained, increased to $40 \mathrm{mcg} / \mathrm{h}$ for the next 4 hours and, if no response persists, increase to $60 \mathrm{mcg} / \mathrm{h}$ for the following 4 hours. He mentions that since the introduction of the described pattern in 2004 in his hospital centre, the cesarean rate has decreased from $13.68 \%$ in 2004 to $6.58 \%$ in 2007.

Based on all these works we can say that it seems that the administration of misoprostol oral solution, administering dosage having in mind uterine response, is associated with a lower cesarean rate and lower incidence of hyperstimulation than using the vaginal misoprostol, gold standard for the preinduction/induction of labour in pregnant women with unripe cervix. Furthermore, being its life span very short, it can be useful in case of uterine hyperstimulation and besides a high percentage of women probably prefer this route to induce delivery than the vaginal version.

Once the misoprostol oral solution-administered in dosage according to uterine response-has proved its effectiveness and its satisfactory safety profile to induce labour in general, we need to check these features in our environment and in specific clinical situations such as gestation in the process of extension or in case of premature rupture of membranes, with a live fetus, situations that represent $60 \%$ of the inductions.

The main objective of this reseach is to compare the efficiency, safety and side effects on the mother and fetus of dosed misoprostol oral solution, depending on uterine response, as this is different for each pregnant woman, compared with the vaginal misoprostol, "gold standard" for preinduction/induction of labour, and doing it in our environment and in specific clinical situations, which represents $75 \%$ of the causes of the inductions, the gestation process of extension (41 weeks) and premature rupture of membranes .

\section{HYPOTHESIS}

Hypothesis: There are differences in the rate of vaginal delivery within the first 24 hours when induced with dosed misoprostol oral solution versus vaginal misoprostol.

\section{OBJECTIVES}

\subsection{Main Objective}

To compare dosed misoprostol oral solution efficiency and safety, compared to the present-day "gold standard" vaginal misoprostol, in our environment and in specific clinical situations: extending gestation processes (41 weeks) and premature rupture membranes. 


\subsection{Specific Objectives}

Primary objectives: Estimating the following parameters: 1) Rate of vaginal births in the first 24 hours. 2) Severe perinatal morbidity rate. 3) Severe maternal morbidity rate.

Secondary objectives: Estimating the following parameters: 1) General cesarean section rate. 2) Hyperstimulation syndrome rate. 3) Delivery rate with thick meconium. 4) Delivery rate when oxytocin is needed. 5) Adverse maternal side effects rate: vomiting, diarrhea or fever. 6) Time interval between first dose and delivery. 7) Women's satisfaction with the route of drug administration.

\section{INTERVENTION}

\subsection{Experimental Group}

Dosed misoprotol oral solution. Misoprostol oral solution in doses of $20 \mathrm{mcg} /$ hour $(20 \mathrm{ml})$, during the first 4 hours. Increase to $40 \mathrm{mcg} / \mathrm{h}$, for the next four hours. Increase 60 $\mathrm{mcg} /$ hour for the next 4 hours. A new dose will be provided, if no correct dynamic were achieved. To prepare the solution, dissolve a $200 \mathrm{mcg}$ misoprostol pill into a in a container with $200 \mathrm{ml}$ of water so that the concentration is $1 \mathrm{mcg} / \mathrm{ml}$. Administer a placebo: vaginal pill each 4 hours.

\subsection{Control Group}

Misoprostol vaginal pill. Compressed $25 \mathrm{mcg}$ vaginal misoprostol every 4 hours until adequate dynamic, maximum dose: 6 pills in 24 hours. Placebo: Oral solution of water with the same dosage as indicated previously.

\section{METHOS/DESIGN}

\subsection{Design}

Double blinded controlled clinical trial. The medication is prepared by the hospital pharmacy. The delivery room staff and pregnant women are unaware of the medication that is being used. Randomization is performed at the time of induction, using sealed opaque envelopes, generated by computer sequence.

\subsection{Scope}

Gynaecologic Clinic Management Unit. Hospital La Inmaculada (Huercal-Overa) that belongs to the Northern Health Management Area from Almeria (Andalusia Health Service). Andalusia (Spain).

\subsection{Reference Population}

The number of users which correspond to the health area is 120,000 . The population is predominantly rural, work- ing primarily in agriculture and the service sector. With a high rate of female migrants/foreigners; the percentage of these in 2010 accounted for $30 \%$ of the new mothers. The main regions/countries of origin are: Morocco, South America and English speaking countries. During 20121216 deliveries were done, being the rate of inductions $21 \%$ and of C-sections $16 \%$. The perinatal mortality rate was 4.81 per thousand. Specifically, the reference population would be pregnant women whose delivery will be treated in our unit.

\subsection{Study Population}

Pregnant women whose labour will be induced due to the fact that they have premature rupture of membranes or a gestation of 41 weeks, cases which represent more than $75 \%$ of our causes for induction.

\subsection{Inclusion Criteria}

Pregnant women whose labour will be induced by any of the following two reasons: premature rupture of membranes or in the process of prolonging gestation (41 weeks) and that meet the following criteria:

Bishop Test for less than or equal to 7. Single Pregnancy. Term gestation in the case of ruptured membranes (37 - 41 weeks). No history of uterine surgery. Cephalic presentation. Live fetus. No contraindications to the use of prostaglandins (asthma, glaucoma or allergies).

\subsection{Exclusion Criteria}

Excluded from the study those pregnant women who have any of the following requirements:

Pregnant women under 18. History of uterine scar. Multiparous with more than 3 deliveries. Multiple pregnancy.

Prior fetal monitoring prior unsatisfactory. Placenta previa. Antepartum genital bleeding when cause of bleeding is unkown. Obstetric Pathology: intrauterine growth retardation (less than or equal to the 10th percentile), ligohydramnios (low amniotic sac less than 2 to 1 ) and hypertensive pregnancy disease. Contraindications for the use of prostaglandins (asthma or glaucoma). Malpresentation: Transverse situation. Breech presentation.

\subsection{Sample Size}

For sample size calculation it has been taken into account the following assumptions: the rate of vaginal births in the first 24 hours when induced with vaginal misoprostol is $59 \%$ and the rate of vaginal birth with dosed oral misoprostol is $71 \%$ (data from the bibliography). The statistical power of the study is $80 \%$ and the confidence level of 5\%. With these data the sample size should be 194 cases. On the other hand it has been calculated an ex- 
pected proportion of losses of $15 \%$, therefore the sample, adjusted to the possible losses, is 229 .

Losses will be due mainly to dropouts, being the main reason that pregnant woman requests a caesarean section, without medical indication there, once induction has been started.

The distribution of pregnant women between the two groups, experimental and control will be held at 1:1 ratio.

\subsection{Dependent and Independent Variables}

Main dependent variables: 1) Vaginal delivery in the first 24 hours. 2) Severe perinatal morbidity. 3) Severe maternal morbidity.

Secondary dependent variables: 1) Form delivery culmination: Vaginal or cesarean. 2) Hyperstimulation syndrome. 3) Presence of thick meconium 4) Adverse maternal side effects: vomiting, diarrhea or fever. 5) Deliveries that require oxytocin. 6) Time interval between the first dose and delivery 7) Women's satisfaction with the route.

Main independent variable: Dosed oral misoprostol use/vaginal misoprostol use.

Secondary independent variables: Maternal age. Gestational age in days. Parity. Body Mass Index. Bishop Score. Length of the Uterine Cervix. Epidural anaesthesia

\subsection{Statistical Analysis}

For the statistical analysis the SPSS software programme for Windows version 17 has been used. First of all a descriptive univariate analysis is done for each treatment group separately. Quantitative variables will be described using the mean, the standard deviation and the range. To obtain qualitative variables, the absolute and relative frequencies expressed in percentages will be used. The differences between quantitative independent variables will be measured by the Student $t$ test or the MannWhitney one, considering significant $p$ below 0.05 . The association between qualitative independent variables with the dependent variable will be checked with the Chi-squared test (with Yates continuity correction where appropriate) or Fisher's exact test. A simple bivariate logistic regression model will be used to measure the existing association between the independent and dependent variable. The possibility of a multiple logistic regression will be considered in order to determine the effect of the independent variables together with the dependent variables. A regression model will be generated for each one of the dependent variables. When the dependent variable considered were quantitative, a multivariate linear regression model, will be used; in both cases the model will be made by introducing statistical signifi- cance variables with a high clinical sense. The treatment of missing data will be made with an intention to treat

\section{ETHICAL RESEARCH}

For informed consent, midwife or obstetrician, explain the pregnant woman the research project and they give her an informed consent form that meets the conditions set out in Law 14/2007 of 3 July Biomedical Research and the Law 15/1999 of December 13, Protection of Personal Data. The form explains the purpose of the research, the intervention to be performed and the results expected from it. It also will ensure the confidentiality of data and anonymity of the participants. If she agrees to participate in the study, she will have to do so by means of a written consent. The informed consent form is also in Arabic and English. The study has been approved by the Coordinating Committee on Ethics in Research of Andalusia.

The researcher is committed to follow the research standards and rules according to the Declaration of Helsinki and subsequent amendments to the 59th General Assembly, Seoul, Korea, October 2008, and to report adverse reactions as provided in RD 1344/2007 of 11 October. Likewise, attendance of pregnant women will be held under the rules of good clinical practice.

\section{DISCUSSION}

Nowadays induction rates are very high, ranging from $25 \%$ to $30 \%$ approximately. In these cases caesarean rates are higher than when the delivery starts spontaneously, the rate almost double. That is one of the main reasons why caesareans have increased, mainly in the cases of nuliparous women with immature cervix. The increase in the number of cesarean deliveries causes among mothers: higher levels of morbidity, higher incidence and risk of nosocominial infections, higher needs of blood transfusions, a much slower pace of recovery; among new-born babies: lower levels of breast feeding, higher frequency of respiratory distress, compared to the normal vaginal delivery.

On the other hand, the rate of nuliparous women is increasing dramatically. The percentage is above $50 \%$ nowadays.

If we can prove the hypothetical good results obtained through the use of dosed oral misoprotol, we will be able to reduce the number of induced deliveries by cesarean, and so improve the levels of security for the mother and the foetus, and, as a consequence, provide a higher quality of medical attention to the new born and the mother.

\section{AUTHORS' CONTRIBUTIONS}

LA conceived the project and all the authors contributed to design of the study. LA, MS and MG initially drafted 
the protocol and all authors were involved in critical revision of the intellectual content. VG, RF and AB obtained ethics approval for the trial. LD and ER negotiated access to population health data for outcome assessment. All authors approved the final protocol.

\section{REFERENCES}

[1] J. Zhang and W. F. Raiburn, "Rising Rates of Labour Induction: Present Concerns an Future Strategies,” $\mathrm{Ob}$ stetrics \& Gynecology, Vol. 100, No. 1, 2002, pp. 164167 http://dx.doi.org/10.1016/S0029-7844(02)02047-1

[2] X. Zhang, K. S. Joseph and M. S. Kramer, "Decreased Term and Postterm Birthweight in the United Sates: Impact of Labour Induction," American Journal of Obstetrics \& Gynecology, Vol. 203, No. 2, 2010, pp. 124.e1124.e 7

[3] C. S. Ennen, J. A. Bofill, E. F. Magnann, J. D. Bass, S. P. Chauhan and J. C. Morrison, "Risk Factors for Cesarean Delivery in Preterm, Term and Post-Term Induction of Labour Patients Undergoing with an Unfavorable Cervix," Gynecologic and Obstetric Investigation, Vol. 67, No. 2, 2009, pp. 113-117.

http://dx.doi.org/10.1159/000166307

[4] D. B. Ehrenthaln, X. Jiang and D. M. Strobino, "Labour Induction and the Risk of Cesarean Delivery among Nulliparous Women at Term," Obstetrics \& Gynecology, Vol. 116, No. 1, 2010, pp. 35-42 http://dx.doi.org/10.1097/AOG.0b013e3181e10c5c

[5] W. F. Rayburn, "Minimizing the Risks from Elective Induction of Labour,” Journal of Reproductive Medicine, Vol. 52, No. 8, 2007, pp. 671-676

[6] F. P. Vrouenraets, F. J. Roumen, C. J. Dehing, E. S. Van den Akker, M. J. Aarts and E. J. Scheve, "Bishop Score and Risk of Cesrean Delivery after Induction of Labour in Nulliparous Women,” Obstetrics \& Gynecology, Vol. 105, No. 4, 2005, pp. 688-689. http://dx.doi.org/10.1097/01.AOG.0000152338.76759.38

[7] A. Vahratian, J. Zhang and J. F. Troendle, "Labour Progression and Risk of Cesarean Delivery in Electively Induced Nulliparous,” Obstetrics \& Gynecology, Vol. 105, No., 2005, pp. 698-704. http://dx.doi.org/10.1097/01.AOG.0000157436.68847.3b

[8] L. J. Heffner, E. Elkin and R. C. Fretts, "Impact of Labour Induction, Gestational Age and Maternal Age on Cesarean Delivery Rates,” Obstetrics \& Gynecology, Vol. 102, No. 2, 2003, pp. 287-293.

http://dx.doi.org/10.1016/S0029-7844(03)00531-3

[9] G. J. Hofmeyr, A. M. Gulmezoglu and C. Pileggi, "Vaginal Misoprostol for Cervical Ripening and Induction of Labour," Cochrane Database of Systematic Reviews, Vol. 10, 2010, Article ID: CD000941.

[10] S. C. Austin, L. Sanchez Ramos and C. D. Adair, "Labour Induction with Intravaginal Misoprostol Vaginal Insert Compared with Dinoprostone: A Systematic Review and Meta-Analysis," American Journal of Obstetrics \& Gynecology, Vol. 202, No. 6, 2010, pp. 624 e1-e9.

[11] R. Shakya, J. Shrestha and P. Thapa, "Safety and Effi- cacy of Misoprostol and Dinoprostone as Cervical Ripening Agents,” Journal of Nepal Medical Association, Vol. 49, No. 177, 2010, pp. 33-37

[12] D. B. Silfeler, B. Tandogan, H. Ayvaci, I. Silfeler, I. Yenidede and V. Dayicioglu, "A Comparison of Misoprostol, Controlled-Release Dinoprostone Vaginal Insert for Cervical Ripening and Oxytocin,” Archives of Gynecology and Obstetrics, Vol. 284, No. 6, 2011, pp. 13311337. http://dx.doi.org/10.1007/s00404-011-1844-7

[13] A. Elati and A. D. Weeks, "The Use of Misoprostol in Obstetrics and Gynecology,” BJOG, Vol. 116, Suppl. 1 2009, pp. 61-69. http://dx.doi.org/10.1111/j.1471-0528.2009.02329.x

[14] A. E. Bartuservicius and R. Nadisauskiene, "Barcaite Oral, Vaginal and Sublingual Misoprostol for Induction of Labour," International Journal of Gynecology \& Obstetrics, Vol. 91, No., 2005, pp. 2-9. http://dx.doi.org/10.1016/j.ijgo.2005.07.002

[15] A. Goldberg, B. S. Greenberg and P. Darney, "Misoprostol and Pregnancy," The New England Journal of Medicine, Vol. 344, No. 1, 2001, pp. 38-47. http://dx.doi.org/10.1056/NEJM200101043440107

[16] ACOG Committee on Obstetric Practice, "ACOG Committee Opinion Number 283, May 2003: New U.S. Food and Drug Administration Labelling on Cytotec (Misoprostol) Use and Pregnancy,” Obstetrics \& Gynecology, Vol. 101, No. 5, 2003, pp. 1049-1050. http://dx.doi.org/10.1016/S0029-7844(03)00396-X

[17] Z. Alfirevic and A. Weeks, "Oral Misoprostol for Induction of Labour," Cochrane Database of Systematic Reviews, Vol. 2, No., 2006, Article ID: CD0001338

[18] R. U. Khan, H. El Rafaey, S. Sharma, D. Sooranna and M. Stafford, "Oral, Rectal and Vaginal Pharmacokinetics of Misoprostol,” Obstetrics \& Gynecology, Vol. 103, 2004, pp. 866-870.

http://dx.doi.org/10.1097/01.AOG.0000124783.38974.53

[19] M. C. Williams, J. C. Tsibris, G. Davis, J. Baiano and W. F. O’Brien, "That Variation Dose is Associated with Approximated One-Quarter Tablet Doses of Misoprostol," American Journal of Obstetrics \& Gynecology, Vol. 187, No. 3, 2002, pp. 615-619. http://dx.doi.org/10.1067/mob.2002.124959

[20] G. J. Hofmeyr, Z. Alfirevic, B. Matonhodze, P. Brocklehurst, E. Campbell and V. C. Nikodem, "Titrated Oral Misoprostol Solution for Induction of Labour: A MultiCenter, Randomized Trial,” BJOG, Vol. 108, No. 9, 2001, pp. 952-959.

[21] Y. Chong, S. Chua, L. Shen and S. Arulkumaran, "Does the Route of Administration of Misoprostol Make a Difference? The Uterotonic Effect and Side Effects of Misoprostol Given by Different Routes after Vaginal Delivery," European Journal of Obstetrics \& Gynecology and Reproductive Biology, Vol. 113, No., 2004, pp. 191-198. http://dx.doi.org/10.1016/j.ejogrb.2003.09.011

[22] O. S. Tang, H. Gemzell-Danielsson and P. C. Misoprostol, "Pharmacokinetic Profiles, Effect on the Uterus ans Side-Effects," International Journal of Gynecology \& Obstetrics, Vol. 99, No., 2007, pp. S160-S167. http://dx.doi.org/10.1016/j.ijgo.2007.09.004 
[23] M. Zieman, S. Fong, N. D. Benowitz and P. Darney, "Banskter Absorption Kinetics of Misoprostol with Vaginal Ortal or Administration,” Obstetrics \& Gynecology, Vol. 90, No. 1, 1997, pp. 88-92.

http://dx.doi.org/10.1016/S0029-7844(97)00111-7

[24] S. Y. Cheng, H. Ming and J. C. Lee, "Compared with Vaginal Oral Titrated Misoprostol for Labour Induction: A Randomized Controlled Trial," Obstetrics \& Gynecology, Vol. 111, No. 1, 2008, pp. 119-125. http://dx.doi.org/10.1097/01.AOG.0000297313.68644.71

[25] T. W. Kundodyiwa, Z. Alfrevic and A. D. Weeks, "LowDose Oral Misoprostol for Induction of Labour," Obstetrics \& Gynecology, Vol. 113, No. 2, 2009, pp. 374-383.
[26] A. S. Souza, A. Scavuzzi, D. C. Rodrigues, R. D. Oliveira, F. E. Feitosa and M. M. Armorim, "Titrated Oral Misoprostol Solution of Labour for Induction: A Pilot Study,” Rev Bras Obstetrics \& Gynecology, Vol. 32, No. 5, 2010, pp. 208-213.

http://dx.doi.org/10.1590/S0100-72032010000500002

[27] S. H. Cheng, C. S. Hsue, G. H. Hwang, W. Chen and T. Ch. Li, "Comparison of Labour Induction with Titrated Oral Misoprostol Solution and Multiparous between Nulliparous Women,” Journal of Obstetrics and Gynaecology Research, Vol. 36, No. 1, 2010, pp. 72-78. http://dx.doi.org/10.1111/j.1447-0756.2009.01118.x 